\title{
Is Comparison Based on Translatable Formal Concepts?
}

\author{
Kevin Guilfoy \\ Philosophy Department, Carroll University, Waukesha, WI 53186, USA; kguilfoy@carrollu.edu
}

Received: 17 December 2019; Accepted: 11 March 2020; Published: 1 April 2020

\begin{abstract}
Fully realized ethical and metaphysical concepts have intension and extension only within an historically situated epistemic tradition. Only people who live the epistemic tradition fully understand the concept and can accurately identify items that satisfy the concept. Such concepts are only fully understood by those whose lives are shaped from within the epistemic tradition. This makes comparison of ethical and metaphysical concepts across epistemic traditions difficult if not impossible. Comparative hagiology employs theological concepts that may function differently from ethical and metaphysical concepts. The articles in this volume seem to suggest that some theological concepts may function as formal concepts. A formal concept is defined by rules or form, rather than by its intensional or extensional content. Thus, formal concepts may be translatable across epistemic traditions. Because the rules do not fully determine intension or extension, a formal concept can apply to otherwise diverse individuals. Theological concepts may be formal concepts that could provide the basis for comparison of the untranslatable concepts that give meaning and value to the lived experience of people in epistemic traditions. The articles in this volume suggest several candidates for such formal concepts.
\end{abstract}

Keywords: comparative method; comparative religions; formal concepts; hagiography; hagiology; sainthood; theory and method in religious studies; translation

I have read the articles in this volume as an outsider. I am not a member of any religious tradition or community, nor am I steeped in the scholarly community of comparative hagiology. While this makes me reticent to offer commentary, Todd French argues that the inclusion of outside scholarly perspectives can aid the discussion (French 2019). My recent work has been on the translation of ethical concepts between traditions, not the comparison of concepts across traditions. Translation in the context in which I work means taking the concept epistemic tradition and claiming that there is a fully synonymous concept in another. In my work, I have argued that ethical and metaphysical concepts are historically situated, socially constructed, and are only meaningful within an epistemic tradition (Guilfoy 2018). The concept of "saint" cannot be carried over into a non-Christian tradition or applied in a non-Christian tradition. The concept of saint only has meaning and content as culturally and historically situated within the Christian epistemic tradition. Such a tradition defines the world and what exists in it, sets the standards for epistemic practice, and provides a framework of meaning and value for people's lived experience. There literally cannot be a saint outside the tradition in which the concept is defined, because the epistemic tradition defines the concept for that tradition from within that tradition. This is not a conclusion I am happy with. One scholar has argued that as a result, there can be no meaningful cross-cultural conversations. ${ }^{1}$ Translation between traditions may be impossible. The articles in this volume and the conference workshop have given me hope that comparison is not.

1 Rowe (2016), develops and defends this concept of translation. See especially chapter 7 and 8 . I doubt I apply the concept with the same intension as he does. Like all excellent philosophical arguments, Rowe leads one through a series of reasonable 
An example from my own college experience illustrates both what I mean by translation and the difference I see between translation and comparison. My experience in comparative religion ended in the 1980s when a professor asserted that the Great Spirit was like the Judeo-Christian God, just less fully developed and understood by Native Americans. This seemed absurd and more than a little racist. Thus, this college freshman never took another religious studies class.

My professor attempted translation not comparison. She attempted to apply, without modification, the ethical, theological and metaphysical concepts of one tradition into another. My professor looked at a different tradition to find an analogue for a key concept in her own. Finding something similar enough, she declared the differences to be the result of their inadequate understanding of her concept. At best, translation creates a shallow and trivial grasp of cultures and traditions. ${ }^{2}$ At worst, it is imperialistic. The discomfort authors in this Special Issue have with cross-cultural application of the concept "saint" shows that it is an historically situated and culturally defined concept that cannot be translated into non-Christian traditions. ${ }^{3}$ I worry that I am doing the same thing in this comment. In what follows, I suggest that the underlying problem can be understood as a philosophical problem-a problem of conceptual translation. With all the arrogance of a philosopher, I suggest that the philosophical concept of a formal concept might help religious studies scholars to better understand their own project.

The argument that translation is impossible is simple enough to outline. The versions I'm most familiar with were developed from the logic and philosophy of science in the last century. ${ }^{4}$ Quine argued that kind terms naming physical objects are not translatable between cultures. When a German points at an object and says "katze," there is no guarantee that the German's ontology includes cats as I understand cats. In the strongest form of Quine's argument, even concepts that apply by ostension to items we can experience via the senses cannot be translated between epistemic communities (Quine 1969). The German's ontology can be radically different from my own but phenomenologically indistinguishable. She may literally be pointing at a different object from the one I see. Ontology is relative to epistemic tradition. Ethical, theological, and metaphysical concepts are not reducible to shared sensory experience. Their meanings are socially constructed and historically situated. The conceptual frame form for identifying an object that satisfies an ethical or metaphysical concept is more complex and, because it is evaluative, cannot be reduced to sensory experience. "Saint" is such a concept. It is not merely an evaluative ethical concept, it is a concept that also includes a vast amount of culturally created value and meaning that shapes the lived experience of those in the Christian tradition. Saints literally do not exist in other epistemic traditions.

I find something comparable to this line of thought in all the articles in this volume. Of course, as an outsider, I cannot be sure that any of the authors understand the project as I do. ${ }^{5}$ Each of the authors attempts to avoid the pitfalls of translation. French describes a method of collaborative checks

and well-argued steps to a counter intuitive and controversial conclusion: it is not possible to have meaningful ethical discussions across traditions. Translation by analogy, or connotation, or vagueness, may be possible, but this is beyond the scope of this reply. Rowe cites Alastair Macintyre as providing a potential model for one tradition to compare and learn from another (MacIntyre 1990). Comparison is possible when an individual in one culture learns another as a "second Language." However, comparison, as Rowe describes it, is the evaluative, even judgmental comparison that is ethically problematic (Rowe 2016).

2 There are advantages to trivial understanding (see Stout 1988). Ineffable metaphysical issue spawn irresolvable disputes. Many have died over an iota of difference. Acknowledging superficial similarities and ignoring unanswerable disputes about metaphysics can encourage tolerance. On the other hand, it is precisely those ineffable metaphysical issues that give meaning and value to people's lives.

3 Many in this volume have responded favorably to Keune's use of "hagio-" rather than "saint-" "Hagio-" is a likely candidate as a formal concept, one that can allow for cross cultural comparison (Keune 2019). I believe this is why Keune's suggestion has been favorably received. In order to focus on the problems using untranslatable concepts, I will continue to use "saint" and its variants.

4 There are other versions that developed in political theory (Hayek 2011; Gaus 2016) and in the post-modern tradition.

5 I would like to thank an anonymous reviewer for demonstrating that translation itself is an untranslatable concept between various academic disciplines. I hope I have presented my discipline's version of the concept clearly. The word "translation" is the same across disciplines. However, the concept signified by the word is quite different. Thus, the items identified as satisfying that concept within one discipline may not be identified as satisfying the concept within another. At best, there are confusing usages of the word "translate" to indicate analogous concepts. 
to insure that one is not translating concepts between cultures (French 2019). This collaborative model could produce an understanding of how ethical, theological, and metaphysical concepts function in different cultures. If we are careful, perhaps, we can compare the forms and function of patronage and veneration exhibited by particular individuals in different epistemic traditions and thus, better understand the many forms of patronage and veneration. There are limits. We cannot know the meaning and value of what we label veneration and patronage in the lived experience of the people engaged in the practices we compare. There is also the risk that a group of scholars actively engaged to insure that ethical, metaphysical, and evaluative terms are not misapplied from one culture to another, find themselves with literally nothing to talk about.

The other contributions to this volume point towards a reliance on formal concepts as a way to ground meaningful cross-cultural comparison. A formal concept is a concept defined by rules or form, rather than by its intensional or extensional content. Formal concepts are not uncommon. "Good" may seem like an untranslatable ethical concept. What is identified as "good" varies radically across epistemic traditions. The precise intensional and extensional content of one tradition's concept of "good" cannot be translated into a different cultural and epistemic context. Nonetheless, we are quite comfortable treating "good" as a formal concept. X is understood as good in one tradition. $\mathrm{Y}$ is understood to be good in another. We can delineate a set of observable "behaviors, practices, beliefs and productions" that allow us to identify different conceptions of the good in other epistemic traditions (Rondolino 2019). We can observe and compare the way a fully realized concept "good" functions in different traditions.

To explain my point, I am going to focus on Rondolino's contribution. Yet, the other contributors are equally suggestive. Keune questions the value of fully stipulative definitions and suggests that we "step back and reconsider whether it truly is the case that we must clearly define our key terms before proceeding with comparison" (Keune 2019). Ritchey's list of concepts contains several that could be formal concepts (Ritchey 2019). There is a way of constructing taxonomies with formal concepts in the subaltern categories. DeValerio's taxonomy could be developed as a nominalist tree of porphyry (DiValerio 2019). That is, with rules that determine several distinct sets of individuals that satisfy the formal concept "perfected." There would be compassionate, sacrificial, and spiritual, species of "perfected" but not simply perfected beings. ${ }^{6}$

In Rondolino's argument hagiography could easily be a formal concept. (Rondolino 2019). Rondolino suggests we jettison the concept "saint" as too culturally specific, and adopt a definition of hagiography that rests on an embedded concept of "perfection." " In the spirit of DiValerio's argument, I suggest that he could build a formal taxonomy of perfection. There are multiple fully realized concepts of who is perfected in different traditions. Each tradition will have its own metaphysical beliefs about what a person is, flaws or shortcomings, the path to fixing those flaws, and ultimately, what a person would look like flawless. Each fully realized concept of perfection is only meaningful within a tradition. A saint is a person who has achieved perfection as understood in the Christian tradition. The perfected Hindu would not have the qualities, actions, etc., that define value and provide meaning to a person in the Christian tradition. A person perfected in the Hindu tradition would not satisfy the concept "saint." By clarifying the rules that allow us to identify individuals recognized as perfected, we can do comparative hagiology. However, is "perfection" a formal concept that would allow comparison?

"Perfection" is clearly central to Rondolino's re-definition of hagiography: "The complex web of behaviors, practices, beliefs and productions (literary, visual, acoustic, etc.) in and by which a given

6 In a nominalist tree of Porphyry, only the lowest level of the taxonomy specifies individuals. There are foxes, rats, and ants, but strictly speaking, no animals. A fox satisfies the rules for being called an animal, but is only fully realized as a fox. To paraphrase Peter Abelard, only discreet individuals exist, but there are rules governing where they fall into categories.

7 One of the rules governing the application of the formal concept hagiography is that the individual who satisfies the concept be "perfected." 
community constructs the memory of individuals who are recognized as the embodied perfection of the "religious" ideal promoted by the community's tradition and socio-cultural context" (Rondolino 2019).

Rondolino's perfection is questionable. Does the concept of "perfection" have rules and a form sufficient to be satisfied in radically different, perhaps mutually exclusive ways in different traditions? Is there a cross-culturally identifiable form, function, or structure of "perfection" that can identify (most of) the radically different individuals identified in different traditions as perfect?

The first clause of Rondolino's definition describes observable behavior, (provided we take reports of beliefs as behavior.) The problem is in identifying any set of behaviors as constructing the memory of individuals recognized as embodied perfection. "Good" works as a formal concept because the behaviors indicative that something has satisfied the rules for applying the concept are fairly clear and universal. ${ }^{8}$ The concept of "Perfection" and the behaviors that would indicate a response to perfection seem more complex and irreducibly culturally situated. Only those living an epistemic and ethical culture can truly understand what perfection is, why it is perfection, what is being perfected, etc. The behavioral responses to "perfection" can only arise in a tradition that has a well-developed concept of the perfect. The observable behaviors people engage in to construct the memory of the perfected individual are themselves socially constructed. The meaning of the behaviors is established within the lived experience of those whose lives are shaped by the tradition. I doubt there is a way to identify a culture's behavioral responses to perfection without translating our concept perfection into that culture. "Perfection" may be an untranslatable concept.

Ritchey's example of Christina makes me think these are more than just academic worries. "Although her (Christina's) devotional behavior struck her contemporaries as positively aberrant-at one point, she is described as climbing into treetops to pray while rolling herself into a ball like a hedgehog (ericei) - Christina was clearly revered by her contemporaries" (Ritchey 2019). Christina may have been revered, but she was also viewed as "aberrant" and "not to be imitated." I do not see a set of rules governing a formal concept of "perfection" that would be satisfied by Christina and Francis. It seems that any observable behavior can be identified as a culturally created response to a perfected individual. We have not left medieval Europe yet.

Ritchey's suggestion of "extra-ordinary" is promising for two reasons. "Extra-ordinary" does not have potentially untranslatable concepts embedded in its definition. Each epistemic tradition will have different understanding of what is ordinary and what is extra-ordinary. This binary is much more fundamental to basic issues of ethics and metaphysics than "perfection." It should be easier to identify what an epistemic tradition deems ordinary and extra-ordinary than it would be to identify what it deems perfect. ${ }^{9}$

"Extra-ordinary" may be too genic for the discipline of comparative hagiology. Rondolino's definition of hagiography also includes the embedded concept of "religion." This seems perfectly reasonable in the context. But untranslatable. There is an ongoing controversy in philosophy. Is Buddhism philosophy or religion? ${ }^{10}$ As I see it, the question involves a category mistake. The concepts "philosophy" and "religion" are historically situated concepts that provided meaning in a certain epistemic tradition. The question translates the concepts into a tradition where they cannot possibly apply. "Extra-ordinary" as a formal concept avoids this issue. This makes "extra-ordinary" much more useful as a formal concept, but runs the risk remove the intellectual core of comparative hagiology.

I am worried that, at best, this brief comment has simply illustrated the problems of applying the conceptual scheme and epistemic process from one discipline to another. I have suggested that the

8 I noted above that we are comfortable treating "good" as a formal concept. Largely, this is because we can track fairly basic human behaviors, attraction and aversion, etc. Attraction and aversion responses are indicative of what a person will judge to be good and bad. This does not give us a very deep understanding of different conceptions of the good, only a way to identify them. This analysis starts with Hobbes, but is also the basis of Moral Foundations Theory (Haidt 2012).

9 I must admit I am imagining a sort of universal look of confusion as the primary indication of the "extra-ordinary."

10 The battle is usually joined between departments that don't want responsibility for teaching material outside their cultural comfort zone. 
concept of a formal concept may be translatable across epistemic traditions. Such formal concepts could provide the basis for comparison of the richer, more fully developed, and untranslatable concepts that give meaning and value to the lived experience of people in epistemic traditions. I am also keenly aware that I may have just repeated the error of my only religious studies professor and demonstrated all the complexities inherent in comparative and interdisciplinary work. I have employed my limited understanding of your project. You seem to be engaged in a form of analysis I can understand from within my own discipline. With all the arrogance of a philosopher, I have suggested a way you can meet the standards of my field. This makes me deeply uncomfortable. There are many fields where the response to such an incursion would be unprintable. This discomfort may be necessary. The authors in this volume have suggested several procedures and guidelines for ethical comparative hagiology. Regardless of how good the procedures and guidelines are, success depends on humility, openness, respect, and above all good will among the contributors to the discussion.

Funding: This research received no external funding

Conflicts of Interest: The author declares no conflict of interest.

\section{List of Contributions}

DiValerio, David M. 2019. A Preliminary Controlled Vocabulary for the Description of Hagiographic Texts. Religions 10: 585. doi:10.3390/rel10100585.

French, Todd E. 2019. Saints across Traditions and Time Periods: Methods for Increasing Range and Reading in Comparative Frameworks. Religions 10: 577. doi:10.3390/rel10100577.

Keune, Jon. 2019. Comparative vs. Hagiology: Two Variant Approaches to the Field. Religions 10: 575. doi:10.3390/rel10100575.

Ritchey, Sara. 2019. Dialogue and Destabilization: An Index for Comparative Global Exemplarity. Religions 10: 569. doi:10.3390/rel10100569.

Rondolino, Massimo A. 2019. Some Foundational Considerations on Taxonomy: A Case for Hagiography. Religions 10: 538. doi:10.3390/rel10100538.

\section{References}

Gaus, Gerald. 2016. The Tyranny of the Ideal. Princeton: Princeton University.

Guilfoy, Kevin. 2018. Peter Abelard's Dialogues: Negotiation is not Translation. Perspectives in Religious Studies 45: $443-56$.

Haidt, Jonathon. 2012. The Righteous Mind. New York: Random House.

Hayek, Friedrich A. 2011. The Constitution of Liberty. Chicago: University of Chicago.

MacIntyre, Alasdair. 1990. Three Rival Versions of Moral Enquiry. Notre Dame Indiana: Notre Dame University Press.

Quine, Willard Van Orman. 1969. Ontological Relativity \& Other Essays. New York: Columbia University.

Rowe, C. Kavin. 2016. One True Life. New Haven: Yale University Press.

Stout, Jeffery. 1988. Ethics after Babel: The Languages of Morals and Their Discontents. Boston: Beacon.

(C) 2020 by the author. Licensee MDPI, Basel, Switzerland. This article is an open access article distributed under the terms and conditions of the Creative Commons Attribution (CC BY) license (http://creativecommons.org/licenses/by/4.0/). 